\title{
Differences between married and unmarried men and women in the relationship between perceived physical health and perceived mental health
}

\author{
John Palner and Maurice B. Mittelmark \\ Research Centre for Health Promotion (HEMIL-senteret), University of Bergen, Bergen, Norway \\ Correspondence to: John Palner, HEMIL-senteret, Christiesgate 13, NO-5015 Bergen, Norway \\ Telephone: +4755588988Ｅ-mail: John.Palner@psyhp.uib.no
}

\begin{abstract}
Married and unmarried men and women were compared with regard to the relationship between perceived physical health and perceived mental health (well being, anxiety and depressive symptoms). Perceived physical health were assessed by self-reported number of medical diagnoses, pain in upper body and in lower body, and physical symptoms such as digestive problems. The study sample was population-based, selected from 13.662 community-dwelling participants in the 1995-96 national health survey conducted by Statistics Norway. Selected were all 275 unmarried men and 271 unmarried women ages 35-67, and equally sized, randomly selected comparison groups in the same age range. A main effect of marital status was observed for all measures of perceived physical health. Married people reported significantly better mental health than unmarried people at all levels of perceived physical health $(\mathrm{p}<0.000)$. Possible buffering effects (stronger protective effect of marriage in the presence of poor perceived physical health) were investigated, but not observed. These results are the first in a community sample in Norway to confirm consistent protective effects (although in cross-sectional data) of marriage.
\end{abstract}

${ }^{1}$ This paper is based on a master's thesis completed by the first author under the direction of the second author, at the Research Centre for Health Promotion. Both authors contributed to the writing of this paper.

\section{INTRODUCTION}

This paper reports on comparisons between married and unmarried Norwegian men and women with regard to the relationship between perceived physical health (number of diagnoses, upper and lower body pain, and physical symptoms) and perceived mental health (well being, anxiety and depressive symptoms). It is a common finding that people who experience multiple health complaints and pain have also poorer psychological health, often in the form of depressive symptoms $(1,2)$. The nature of the relationship between poor physical health and poor mental health is most likely one of reciprocal influence (3).

Besides the personal burden and distress associated with many common health complaints, the costs to society are well documented and substantial. In Norway, musculoskeletal problems in the general population are the most common cause of visiting a general practitioner, the most common cause of absenteeism from the work place, and the trends are worsening $(4,5)$. In 1997, more than 50 percent of all cases of mediumterm absenteeism (three days to two weeks) from the work place were due to musculoskeletal problems (6). Also musculoskeletal problems account for an increasing proportion of early retirement. In 1980, 26 percent of all women and 18 percent men receiving early re- tirement were diagnosed with muscoloskeletal problems. In 1997, the figures had increased to 39 percent for women and 25 percent for men, respectively (4).

At the same time that the disturbing trends cited above are occurring, important social resources that help one cope with chronic health stress may be less abundant in modern society. In Norway as elsewhere, the ageing of the population is accompanied by shrinking social networks, a mobile workforce results in scattered families, and changing familial norms means fewer enduring relationships such as those found in many marriages (5). Indeed, data from Norway indicate fewer people getting married, the proportion of single households increasing and a growing tendency for the Norwegian population to live alone, at least for some parts of their adult lives (5).

The significance of these trends to the population's health may be substantial. There is a large literature showing that persons with chronic diseases experience better physical and mental health outcomes if they have a good social network (7-11). The health protection effect of having a spouse is an especially robust finding (12-14). For social networks in general and for spouse support in particular, two types of effects have been observed. 'Main' effects refers to protective effects that are exerted regardless of level of health. 'Buffer' effects refer to protective effects that are more 
evident when health is poor, and modest or not present when health is good.

Most of the health research on the main and buffering effects of social network has focused on morbidity and mortality associated with major diseases such as the cardiovascular diseases and cancers. The very few studies that have focussed on common health problems have limitations that call for additional research. Jackson (15), for example, observed a buffering effect of support from spouse and friends on the relationship between self-reported physical health problems and depressive symptoms. A critical limitation of the study was its restriction of the sample to married people only. Similarly, Barstad (16) observed a moderating effect of having a confidant on the relationship between self reported health complaints and symptoms of depression and anxiety. In this study as well, the possible influence of marital status was not investigated.

The rationale and design of the present study, which is a secondary analysis of data from the study "Helseundersøkelsen-95", was founded on the observations discussed above. Perceived physical health was hypothesised to be associated negatively with perceived mental health. Marriage was hypothesised to exert both main and buffer effects on the perceived physical/mental health relationship. That is, it was expected that married men and women, compared to unmarried people, would report lower levels of mental health at all levels of physical health. It was expected also that this effect would be more pronounced among those with poor perceived physical health. Further it was expected that the protective effect of marriage would be observed after controlling statistically for other factors presumed to be correlated with both marital status and health status, especially socioeconomic status.

\section{METHODS}

"Helseundersøkelsen-95" was a cross-sectional survey conducted in Norway from September to December, 1995, by Statistic Norway. The study sample was population-based and included a total of 13,662 participants. Information on respondents' perceived health, social network and family relations, occupational information, self-reported utilisation of health care services and information on demographic variables was obtained by interview and by questionnaire. Information was collected also on self-assessed well being, self-reported number of medical diagnoses, and a 35 item modified version of the Hopkins Symptom Checklist (17) was administered. The survey included several components such that not all respondents received the same questionnaires and interviews.

Of the 2,814 participants ages 35-67, the 546 participants who reported being unmarried (single, widow, separated, divorced) at the time of the survey were selected for the present analysis. A comparison group of the same size was selected at random from among the married participants, matched on gender.
The analysis variables were constructed as follows. With the assistance of a principal components factor analysis with varimax rotation performed on the Hopkins Symptoms Checklist variables, five scales were constructed measuring: 1) depressive symptoms (11 items, Cronbach's alpha $=0.89$ ); 2) anxiety (six items, Chronbach's alpha =0.84), 3) upper body pain (six items, Cronbach's alpha $=0.81$ ); 4) lower body pain, two items, Cronbach's alpha $=0.65)$; 5) physical symptoms, six items, Cronbach's alpha $=0.73)($ Table 1$)$.

Table 1. Mean and standard deviation for health complaints and measures of mental health for married men and women.

\begin{tabular}{|c|c|c|c|c|c|c|}
\hline \multirow[b]{2}{*}{ Variables } & \multicolumn{3}{|c|}{ Married } & \multicolumn{3}{|c|}{ Unmarried } \\
\hline & Mean & S.D. & $\mathrm{n}$ & Mean & S.D. & $\mathrm{n}$ \\
\hline \multicolumn{7}{|l|}{ Health complaints } \\
\hline \multicolumn{7}{|l|}{ Diagnoses } \\
\hline Men & 1.85 & 1.25 & 184 & 1.93 & 1.10 & 180 \\
\hline Women & 1.90 & 1.12 & 185 & 2.27 & 1.33 & 193 \\
\hline \multicolumn{7}{|l|}{ Upper pain } \\
\hline Men & 2.29 & 2.29 & 275 & 2.10 & 2.79 & 275 \\
\hline Women & 2.94 & 3.31 & 271 & 3.57 & 3.64 & 271 \\
\hline \multicolumn{7}{|l|}{ Lower pain } \\
\hline Men & 0.41 & 0.41 & 275 & 0.51 & 1.04 & 275 \\
\hline Women & 0.60 & 1.10 & 271 & 0.64 & 1.20 & 270 \\
\hline \multicolumn{7}{|c|}{ Physical symptoms } \\
\hline Men & 0.98 & 1.59 & 275 & 1.20 & 2.18 & 275 \\
\hline Women & 1.32 & 1.87 & 273 & 1.91 & 2.39 & 271 \\
\hline \multicolumn{7}{|l|}{ Mental health } \\
\hline \multicolumn{7}{|l|}{ Well being } \\
\hline Men & 6.48 & 1.84 & 275 & 5.94 & 1.97 & 275 \\
\hline Women & 6.25 & 1.81 & 271 & 5.82 & 2.10 & 271 \\
\hline \multicolumn{7}{|c|}{ Depressive symptoms } \\
\hline Men & 1.70 & 3.29 & 274 & 2.73 & 4.31 & 275 \\
\hline Women & 2.36 & 4.09 & 268 & 3.16 & 4.48 & 269 \\
\hline \multicolumn{7}{|l|}{ Anxiety } \\
\hline Men & 0.92 & 1.97 & 275 & 1.31 & 2.30 & 275 \\
\hline Women & 1.00 & 1.96 & 271 & 1.42 & 2.23 & 268 \\
\hline \multicolumn{7}{|l|}{ Moderator } \\
\hline \multicolumn{7}{|l|}{ Marital status } \\
\hline Men & -- & -- & 275 & -- & -- & 275 \\
\hline Women & -- & -- & 271 & -- & -- & 271 \\
\hline \multicolumn{7}{|l|}{ Control variables } \\
\hline \multicolumn{7}{|l|}{ Negative impact } \\
\hline Men & 1.38 & 1.05 & 191 & 1.48 & 0.99 & 189 \\
\hline Women & 1.58 & 0.99 & 189 & 1.69 & 1.03 & 196 \\
\hline \multicolumn{7}{|l|}{ Age } \\
\hline Men & 49.58 & 8.77 & 275 & 45.77 & 8.64 & 275 \\
\hline Women & 47.83 & 9.03 & 271 & 46.45 & 8.94 & 271 \\
\hline
\end{tabular}

At the interview the respondents were asked whether they had one or more of 12 chronic diseases with duration longer than six months; nervous diseases and symptoms; neurological diseases; cardiovascular diseases; respiratory diseases; dermatological diseases; musculoskeletal diseases; injuries. A maximum of 10 diagnoses was recorded for each respondent. A sum score of all diagnoses was calculated.

Three questions were summed to create a scale of perceived mental health (Cronbach's alpha $=0.69$ ). These were "Have you been happy and satisfied the past two weeks?", "How much of the time have you felt 
in a good mood and had good energy recently?", and "How do you perceive your own health in general?".

The interview included a question on self-rated negative impact of diseases, conditions and symptoms, worded as follows: "We would like to know how you evaluate the various illnesses and functional limitations you have reported. To what degree do these effect your every day living (all kinds of effects, including pain, anxiety, sleeping problems, exhaustiveness and limitations in what you can do)?" This was used in multivariate analyses to control for severity of illness effects.

Marital status and age were determined from national registry data that were added to the data set immediately after the interviews were completed. For the present study, the categories unmarried, widows, divorced and separated were grouped into the category 'unmarried'. Because of the well documented relationship between socioeconomic status, a variable assessing annual personal income was included as a covariate in the analysis.

\section{RESULTS}

\section{Perceived mental health}

Four analyses of variance were conducted with perceived mental health as the dependent variable, and each of the four measures of perceived physical health treated as fixed variables and dichotomised into high and low groups. Marital status was also treated as a fixed, dichotomous variable. Age, personal income and negative health impact were included in the analyses as covariates. Estimated marginal means was obtained for all predictors. Possible buffering effects (a protective effect of marriage in the presence of poor perceived physical health) were investigated by examining statistical interactions between marital status and the variables of perceived physical health in the analysis.

A main effect of the diagnoses variable was observed $(F=13.57, p<0.000)$, with respondents in the high category of the diagnoses variable having significantly lower perceived mental health scores compared to respondents in the low category of the diagnoses variable. A main effect was observed also for marital status $(\mathrm{F}=14.21 ; \mathrm{p}<0.000)$, with married respondents having higher perceived mental health scores than unmarried respondents. A main effect of annual personal income was observed $(\mathrm{F}=18.48 ; \mathrm{p}<0.000)$. The adjusted $\mathrm{R}^{2}$ for this model was 0.32 .

A main effect of upper body pain was observed $(\mathrm{F}=24.27, \mathrm{p}<0.000)$, with those reporting more pain also reporting lower levels of perceived mental health compared to the low pain group. A main effect of marital status was also observed $(\mathrm{F}=14.21, \mathrm{p}<0.000)$, with married respondents reporting higher levels of perceived mental health than unmarried respondents. A interaction between upper body pain by marital status was observed, however not significant $(\mathrm{F}=3.69$, NS). A main effect of annual personal income was also observed $(\mathrm{F}=17.25 ; \mathrm{p}<0.000)$. The adjusted $\mathrm{R}^{2}$ for this model was 0.33 .
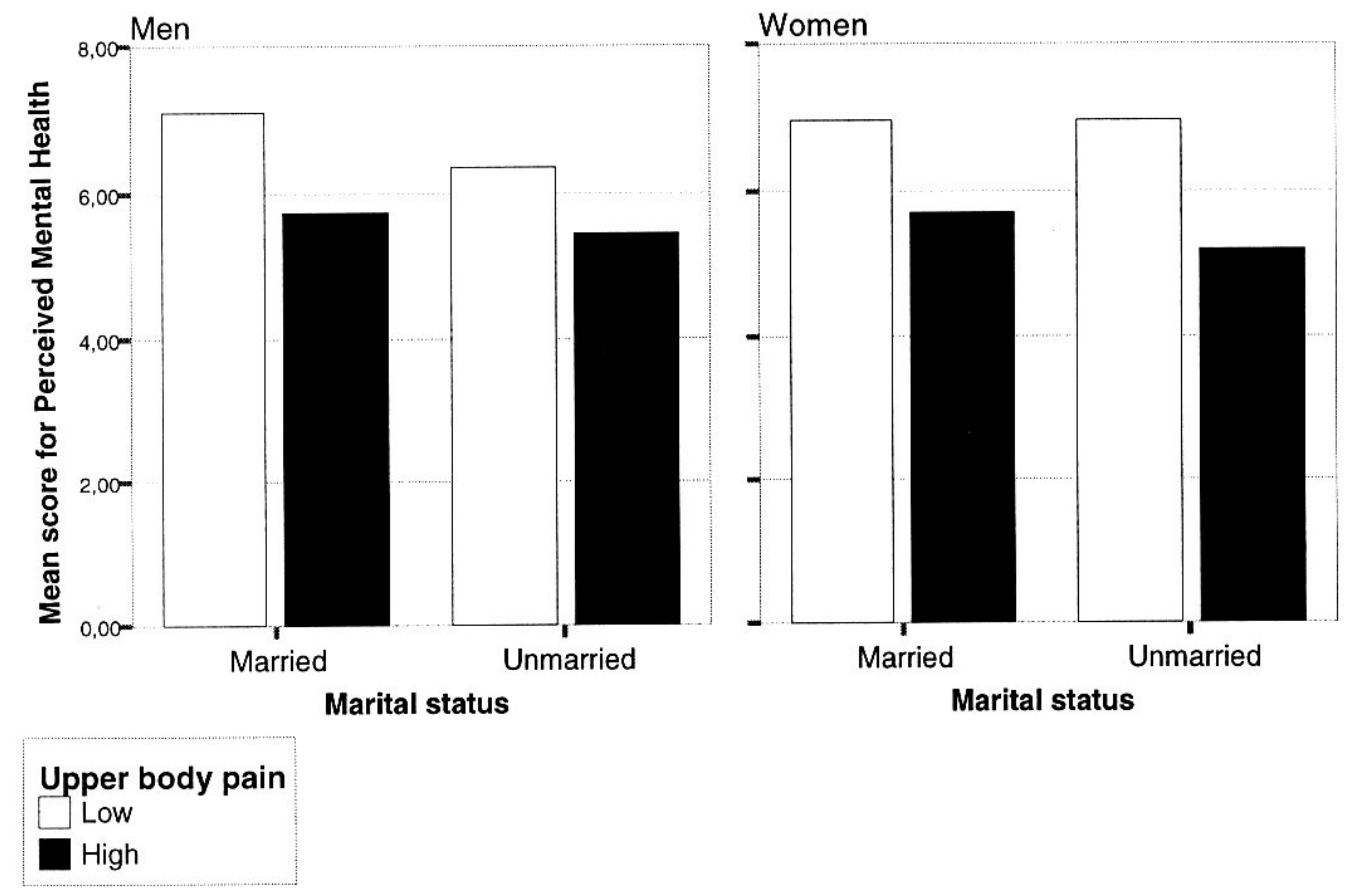

Figure 1. Mean score for perceived mental health, married and unmarried men and women, with low and high upper body pain. 
A main effect of the lower body pain variable was observed $(\mathrm{F}=17.46, \mathrm{p}<0.000)$. Respondents in the high category of the lower body pain variable reporting significantly lower perceived mental health compared to respondents in the low category of lower body pain variable. A main effect was observed also for marital status $(F=16.33, p<0.000)$, with married respondents having significantly higher scores on perceived mental health compared to unmarried respondents. The adjusted $\mathrm{R}^{2}$ for this model was 0.32 .

A main effect of physical symptoms was observed $(\mathrm{F}=60.06, \mathrm{p}<0.000)$, with respondents in the high category of the physical symptoms variable having significantly lower score on the perceived mental health variable, compared to respondents in the low category of the physical symptom variable. A main effect was also observed for marital status $(\mathrm{F}=13.83$, $\mathrm{p}<0.000)$. Unmarried respondents had significantly lower score on the perceived mental health variable, compared to the married respondents. As in the previous analysis, a main effect of income was observed $(\mathrm{F}=12.1, \mathrm{p}<0.001)$ The adjusted $\mathrm{R}^{2}$ for this model was 0.35 .

In the four analyses described above, no statistically significant buffering effects were observed.

The dichotomous depressive symptoms and anxiety variables were analysed with logistic regression. The four dichotomous health stressor variables were used as predictor variables in turn. Other predictor variables included marital status, gender, personal income (in two approximately equal categories), negative health impact (in two approximately equal categories) and age (in two approximately equal categories). Possible buffering effects were investigated by including the interactions of marital status by each health stressor variables in turn.

\section{Depressive symptoms}

A main effect of upper body pain was observed (OR = 2.91, CI: $1.60-5.28, \mathrm{p}<0.000)$ in an analysis in which depressive symptoms was the predicted variable. Respondents in the high category of the upper body pain variable had a significantly higher risk of depressive symptoms, compared to respondents in the low category of the upper body pain variable. A main effect of personal income was observed $(\mathrm{OR}=0.41, \mathrm{CI}$ : 0.30 $0.58, \mathrm{p}<0.000)$. Respondents in the high category of the personal income variable had a significantly lower risk of depressive symptoms, compared to respondents in the low category of the personal income variable. No main effect of marital status was observed, and neither was a buffering effect.

A main effect of the physical symptoms variable was observed (OR $=6.52$, CI: 3.3-12.1, p < 0.000), with respondents in the high category of the physical symptoms variable having a higher risk of depressive symptoms, compared to respondents in the low category of the physical symptoms variable. Also a main effect of personal income was observed $(\mathrm{OR}=0.46$, CI: $0.32-0.66, \mathrm{p}<0.000)$. Respondents in the high category of the personal income variable had a significantly lower risk of depressive symptoms, compared to respondents in the low personal income category. No main effect of marital status was observed, and neither was a buffering effect.

\section{Anxiety}

A main effect of the upper body pain variable was observed (OR $=3.04$, CI: $1.75-5.28, \mathrm{p}<0.000)$, with respondents in the high category of the upper body pain variable having significantly higher risk of anxiety, compared to respondents in the low category of upper body pain. Similarly, a main effect of the physical symptoms variable was observed $(\mathrm{OR}=4.05$, CI: $2.20-7.21, \mathrm{p}<0.000)$. Respondents in the high category of the physical symptom variable had a significantly higher risk of anxiety, compared to respondents in the low category of the physical symptom variable.

\section{DISCUSSION}

The present study has limitations that should be noted from the outset. Because of small sample sizes in the marital status categories separated, divorced, widowed, and never married, these were collapsed into one category of unmarried respondents. However, evidence suggests that mental distress is more prevalent among separated and divorced individuals, compared to married individuals, and this pattern may extend to never married persons as well (18). That these various unmarried subgroups were not studied in their own right is potentially important, since some researchers argue that combining the separated, divorced, widowed and never married into one category may result in an underestimation of the level of mental distress among the separated/divorced persons and overestimation among widowed persons (19).

In this study and some other cross-sectional studies, evidence of a protective effect of marriage on health has been demonstrated. The seeming protective effect of marriage may however be spurious, in part or in whole. It is possible, for example, that good mental health may be more frequently observed among individuals living under more affluent social and material conditions, compared to those that live under relatively poorer conditions, regardless of marital status.

Marital status differentials of health have been observed for a number of socioeconomic indicators such as income, housing tenure and employment. Waite et al. (20) observed a higher per capita income, higher hourly wages and a lower risk for children of dropping out of school among married people compared to cohabiting and divorced, separated and widowed people. In the same study, married people had less alcohol related problems and were less likely to 
engage in negative health behaviour (20).

In Hope et al's investigation of marital transitions and distress (21), housing tenure was observed as an important moderator of the change in mental health in conjunction with marital transition. Poor mental health persisted among married women who were downward mobile in terms of housing tenure compared to those who were upward mobile. The difference was even more pronounced after divorce.

In yet another study, a beneficial effect of marriage on psychosomatic symptoms was observed for women who were unemployed. The differential persisted in each of two successive five-year follow-up intervals. Unemployed women without an alternative source of financial and instrumental support also had the poorest health. Also unemployed women had a higher risk of marital dissolvement compared to employed women (22).

In the present study all exploratory analysis included variables measuring annual personal income, socioeconomic status based on the standard Statistics Norway formulation (23), and educational level. Only annual personal income emerged as significantly associated with the perceived mental health variables. Therefore, personal income was included as a covariate in all the final statistical models presented in this paper. Consistent with many other studies, in almost all the models, a main effect of income was observed. However, protective effects of marriage was evident even when income was included in the models. Thus the marital status health differentials observed are likely due to factors other than societal/macro level factors, a conclusion supported by other analysis of Norwegian data (24).

In this study, a relationship between self-reported common health complaints and mental health measured in different ways was observed. The results are consistent with, and extend, findings from several other similar studies. A similar finding to that reported here, between self-reported health (physical functioning, role limitations due to physical health problems, and satisfaction with ones' own physical abilities) and mental health (anxiety, loss of control, and positive affect), was observed by Sherbourne et al. (25). However, Sherbourne et al.'s sample was restricted to chronically ill patients. This paper generalises the finding, since a broad range of health status was represented in the population-based sample of the present study.

Jackson (15) also observed a relationship between self-reported physical health (perceived health status, perceived physical ability, and perceived health satisfaction), and self-reported depressive symptoms, but the study included only married persons. In the present investigation, since both married and unmarried participants were included, important protective effects of marriage could be investigated, and indeed, were found to be of importance.

More recently, Penninx et al. (26) observed a relationship between self-reported health (such as diabe- tes, cancer, arthritis in knee and hip, or cardiac diseases) and depressive symptoms. The sample consisted of participants aged 55-85 years. The present study, with an age range from 35-67 years, extends the findings to a much younger age group.

Finally, a relationship between physical health (such as functional disability and diagnoses) and mental health (depressive symptoms and anxiety) was also observed by Barstad (16) in a study based on the same data as the present report. In Barstad's analysis, having a confidant influenced the physical health/mental health relationship, but Barstad (16) did not make the marital status comparisons that were the focus of the present investigation.

In this study, married persons, compared to unmarried persons, consistently reported better mental health regardless of their physical health status. This observation is consistent with findings of Walen et al. (27), who found that social exchange from ones' partner was a significant predictor of well being (life satisfaction, positive affect, and negative affect), and also with Sherbourne et al. (25), who observed a main effect of marital status on mental health (depression and anxiety), after controlling for perceived negative health impact and age. The present results are consistent also with the findings of Penninx et al. (26), who observed a main effect of having a partner on the relationship between self-reported arthritis (in knee and hip), and depressive symptoms.

At the same time that main effects of martial status were observed consistently in this study, there was no evidence of buffering effects, and this bears some discussion. Researchers commonly differentiate between two possibilities regarding the health protection effects of social support: Effects than happen only when stress is present (buffering effects) and effects that happen regardless of level of stress (main effects, sometimes referred to as marginal effects). Some authorities in the field hold such strong opinions on the nature of these effects, stated almost as laws; for example, "close relationships have a buffering effect, but networks of friends have a main effect - that is, they work whether stress is present or not" (28).

In fact the situation is more complicated than that. Vilhjalmsson (29), for example, has demonstrated that in community-based studies of life stress, social support and clinical depression, linear multivariate statistical analyses tend to reveal buffering effects, while non-linear analytic approaches tend to reveal main effects in the same data. Variability in analytic outcome may also be influenced by the nature, intensity and duration of the stressor, the investigator's conceptualisation and measurement of support (enacted/ received/perceived), the source(s) of support, the appropriateness or inappropriateness of support attempts, the nature, duration and severity of the health outcome in question, and untold numbers of combinations of these variables.

In the context of the present study, in which stres- 
sors were conceptualised as common health complaints and components of mental health was conceptualised as outcome, both linear multivariate analysis and non-linear analysis was used. Some evidence of a buffering effect of marital status was observed but did not reach statistical significance. This was in part due the fact that the level of significance chosen for this study was set to 0.005 , implying a conservative estimate of probability. Thus 'really' significant results may be discarded in this manner.

Perceived negative health impact (severity) of health stressors was strongly associated with all outcome measures in the present study. It was fortunate that this potential confounder was measured so that it could be controlled for in the multivariate analyses, a step that was not taken in any of the other studies discussed above.

In the literature, some studies have controlled statistically for the effect of gender $(26,16)$ or have not included the variable at all (25). Other studies have examined gender as was done in the present investigation, in which no differences were observed between men and women in the relationship between perceived physical and mental health. This is inconsistent with findings from several other studies indicating that men in general benefit more from marriage than women do, although such findings mostly have been observed in studies of mortality (30) and morbidity (31).

Consistent with the present findings, however, are those of Jackson (15), who observed no differences between men and women in her study on the relationship between self-reported physical health and depressive symptoms. Women have been observed also to benefit more from social support sources outside marriage, such as having (non-spousal) confidant support (27), friendship (32), and material resources (18).

As mentioned in the introduction to this paper, the prevalence of chronic health stressors such as musculoskeletal conditions and mental illness is higher among women compared to men in Norway and elsewhere (33). Therefore, the explicit study of the role of gender in stress and health studies is needed. This study is among the first in Norway to explore the possible differences among men and women on the relationship between perceived physical and mental health.

As discussed above, the literature on the influence of marital status on the relationship between chronic health stressors and mental health is not consistent. One reason for inconsistent findings may be due to the categorising of key variables. As noted by several authors (34) categorising of variables results in loss of information that may consequently limit the ability to detect interaction effects and mask real differences within analysis subgroups. Another plausible explanation for the inconsistency of results in this arena may be that marriage per se is not a resource in times of stress. Intimate relationships may have a dark side (35). This argument is consistent with data from Phennix et al. (26), who observed that receiving instrumental support was associated with more depressive symptoms. Some results from the present study seem to support this possibility. As described earlier, mean score on the perceived mental health variable among married men in the high category of lower body pain approached the score for unmarried men. Lower body pain usually implies functional disability, which in turn may restrain the possibility to participate in social activities and imply a need for instrumental support. Thus, being in need of considerable instrumental support from a partner or feeling socially isolated may cause feelings of helplessness, or dependency, both of which may lead to a decreased level of perceived mental health. However, this conjecture could not be tested in the present study due to limitations in the data.

Concluding with what may be the most obvious point of discussion, because the study used data from a population-based sample, very few people reported high levels of depressive symptoms, anxiety and poor perceived mental health. This resulted in highly skewed distributions with the large majority of participants reporting no or very few problems. Under this circumstance, it seems noteworthy that the protective effects of marriage were observed. Had the sample included a more heterogeneous mix of symptoms, anxiety and perceived mental health, effects might well have been more pronounced.

\section{REFERENCES}

1. Blackburn-munro G, Blackburn-munro R. Chronic pain, Chronic stress and depression: Coincide or consequence? J Neuroendocrinol 2001; 13: 1009-1023.

2. Grossi G, Soares J, Ängeslevä J, Perski A. Psychosocial correlates of long-term sick leave among patients with musculoskelatal pain. Pain 1999; 80: 607-619.

3. Lazarus RS, Folkman S. Stress, Appraisal, and Coping. New York: Springer, 1984: 445.

4. Helsedepartementet. Folkehelserapporten 1996. Oslo: Statens insitutt for folkehelse, 1996.

5. Helsedepartementet. Folkehelserapporten 1999. Oslo, 1999.

6. Rikstrygdeverket. Trygdestatistisk årbok 1998. Oslo, 1998.

7. Berkman L. Assessing the physical health effects of social networks and social support. Annu Rev Public Health 1984; 5: 413-32. 
8. Schwazer R, Leppin A. Social support, health, and health behavior. In: Hurrelmann K, Losel F, eds. Health Hazards in Adolescence: Prevention and Intervention in Childhood and Adolescence. Berlin: Walter de Gruyter, 1990: 528.

9. Cohen S, Kaplan J, Manuck S. Social support and coronary heart disease: Underlying psychological and biological mechanisms. In: Shumaker S, Czajkowski S, eds. Social Support and Cardiovascular Disease. New York: Plenum Press, 1994.

10. Broadwell S, Light K. Family support and cardiovascular responses in married couples during conflict and other interactions. Int J Behav Med 1999; 6: 40-63.

11. Seeman TE. Social ties and health: The benefits of social integration. Ann Epidemiol 1996; 6: 442-451.

12. Ross C, Mirowsky J, Goldsteen K. The impact of the family on health: A decade in review. J Marriage Fam 1990; 52: 1059-1078.

13. Umberson D, Chen M, House J, Hopkins K, Slaten E. The effect of social relationships on psychological well-being: Are men and women really so different? Am Sociol Rev 1996; 61: 837-857.

14. Horwitz A, McLaughlin J, White H. How the negative and positive aspects of partner relationsships affects the mental health of young married people. J Health Soc Behav 1997; 39: 124-136.

15. Jackson P. Specifying the buffering hypothesis: Support, strain and depression. Soc Psychol Q 1992; 55: 363378.

16. Barstad A. Fortrolighet forebygger? Samfunnspeilet 1997; 2: 22-31.

17. Derogatis L, Lipman R, Rickels K, Uhlenhut E, Covi L. The Hopkins Symptom Checklist (HSCL). A selfreport symptom inventory. Behav Sci 1974; 19: 1-15.

18. Joung I, Stronks k, Van de Mheen H, Van Poppel F, Van de Meer J, Mackenbach J. The contribution of intermediary factors to marital status differences in self-reported health. J Marriage Fam 1997; 59: 476-490.

19. Cotten S. marital status and mental health revisited: Examining the importance of risk factors and resources. Fam Relat 1999; 48: 225-233.

20. Waite L. Does marriage matter? Demography 1995; 32: 483-507.

21. Hope S, Rodgers B, Power C. Marital status transitions and psychological distress: longitudinal evidence from a national population sample. Psychol Med 1999; 29: 381-389.

22. Waldron I, Hughes M, Brooks T. Marriage protection and marriage selection - Prospective evidence for reciprocal effects of marital status and health. Soc Sci Med 1996; 43: 113-123.

23. Øien A. Standard for classification of socio economic status. Kongsvinger: Statistics Norway, 1984:32.

24. Elstad J. Inequalities in health related to women's marital, parental, and employment status - a comparison between the early 70s and the late 80s, Norway. Soc Sci Med 1996; 42: 75-89.

25. Sherbourne C, Hays R. Marital Status, Social support and health transitions in chronic disease patients. $J$ Health Soc Behav 1990; 31: 328-343.

26. Phennix B, Tilburg VT, Boeke A, Deeg D, Kriegsman D, Eijk vJ. Effects of social support and personal coping resources on depressive symptoms: Different for various chronic disease? Health Psychol 1998; 17: 551-558.

27. Walen H, Lachman M. Social support and strain from partner, family, and friends: Costs and benefits for men and women in adulthood. $J$ Pers Soc Relat 2000; 17: 5-30.

28. Argyle M. Interpersonal accounts - a social psychological perspective. Br J Soc Psychol 1992; $31: 393$.

29. Vilhjalmsson R. Life stress, social support and clinical depression: A reanalysis of the literature. Soc Sci Med 1993; 37: 331-342.

30. Smith K, Waitzman N. Double jeopardy: Interaction effects of marital and poverty status on the risk of mortality. Demography 1994; 31: 487-507.

31. Shumaker S, Hill D. Gender differences in social support and physical health. Health Psychol 1991; 10: 102111.

32. Antonucci T, Akiyama H. An examination of sex differences in social support among older men and women. Sex Roles 1987; 17: 11-12.

33. Sandanger I, Moum T, Ingebrigtsen G, Sørensen T, Dalgard O. Prevalence, incidence and age at onset of psyciatric disorders in Norway. In Press 1999.

34. Cohen S, Wills T. Stress, social support, and the buffering hypothesis. Psychol Bull 1985; 98: 310-357.

35. Spitzberg B, Cupach W. The Dark Side of Close Relationships. Mahwah: Lawrance Erlbaum Assoc., 1998. 